\title{
Yes, minimally invasive left ventricular device implantation can be done-but should it?
}

\author{
Valluvan Jeevanandam, MD
}

From the Department of Cardiac and Thoracic Surgery, University of Chicago Medicine, Chicago, Ill. Disclosures: V.J. serves as a scientific advisor for Thoratec/St Jude and ReliantHeart.

Received for publication July 11, 2016; accepted for publication July 12, 2016; available ahead of print Aug 21, 2016

Address for reprints: Valluvan Jeevanandam, MD, University of Chicago Medicine, 5841 S Maryland Ave, Rm E500, MC5040, Chicago, IL 60637 (E-mail: jeevan@uchicago.edu).

J Thorac Cardiovasc Surg 2016;152:1448-9

$0022-5223 / \$ 36.00$

Copyright (c) 2016 by The American Association for Thoracic Surgery

http://dx.doi.org/10.1016/j.jtevs.2016.07.021

In their article in this issue of the Journal, Saeed and colleagues, ${ }^{1}$ as others have done, ${ }^{2,3}$ have demonstrated excellent surgical ability by implanting continuous-flow left ventricular assist devices (LVADs) through minimal invasive surgery (MIS) approaches. Smaller intrapericardial devices, such as the HeartWare HVAD (HeartWare, Framingham, Mass) and Thoratec/St Jude HeartMate 3 (Thoratec Corporation, Pleasanton, Calif), facilitate the MIS approaches. They can be performed on or off cardiopulmonary bypass (CPB) and usually involve a left anterior thoracotomy along with a hemisternotomy or right thoracotomy for the outflow anastomosis. The outflow graft can also be anastomosed to the subclavian artery or descending aorta. $\mathrm{CPB}$, if used, requires femoral cannulation.

MIS approaches are really a misnomer. The combination of a thoracotomy for the inflow connection and another incision for the outflow, along with a site for CPB cannulation (Figure 1), neither decreases the length of total incisions nor the postoperative pain relative to the conventional approach. MIS approaches really should be referred to as nonsternotomy approaches.

There have been no randomized trials comparing the nonsternotomy approach with standard implantation. The best comparison has been a retrospective propensity-

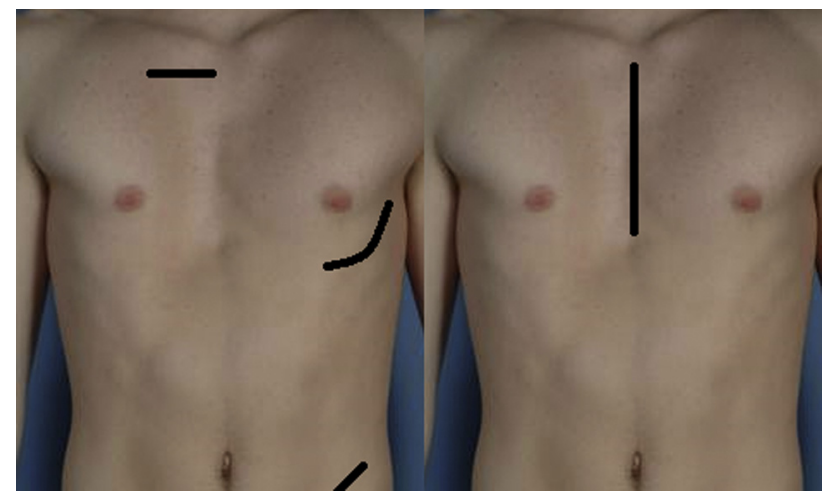

FIGURE 1. Three incisions versus one-is there an advantage? The nonsterntomy approach is on the left and a sternotomy is on the right.

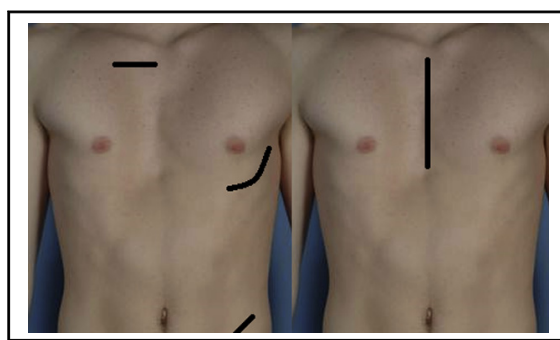

Three incisions versus one-is there an advantage?

Central Message

Miniaturized intrapericardial LVADs can be implanted without a sternotomy. Is there a compelling reason for this to become standard practice, or should it be used only for selected patients?

See Article page 1446.

matched review which did not show a survival benefit. Selection and surgical bias therefore taint the ability to determine superiority of the nonsternotomy technique. There are some advantages in the selected group of patients receiving the nonsternotomy approach. Obviating the full sternotomy could decrease need for blood transfusions. Immediate postoperative RV function seems to be better preserved because heart manipulation and pericardial disruption are minimized. The difficulty of resternotomy for transplant or explant might be decreased. The one clear advantage of the NS approach would be seen in patients with "hostile surgical chests" - patients with patent grafts crossing the midline, the right ventricle adherent to the sternum, multiple sternotomies, and so on. A thoracotomy to implant the LVAD inflow would help avoid the possible complications of a sternotomy, but a proper location for the outflow graft would remain a challenge.

There are disadvantages to the nonsternotomy approach. It hinders adjunctive procedures such as (1) valve repair or replacement (tricuspid, mitral, and aortic); (2) removal of left ventricular thrombus, especially if being done without $\mathrm{CPB}$; and (3) full visualization to ensure proper positioning of the system before closure. It also places the LVAD in the pleural space, with possible direct contamination in patients with pneumonia or empyema, and creates adhesions to the lung, phrenic nerve, and diaphragm, making reoperations more difficult. 
Nonsternotomy implantation of the HVAD and HeartMate 3 are adjunctive options for surgeons implanting LVADs. A true MIS implantation would obviate the need for a sternotomy or thoracotomy, eliminate the need for a long rehabilitation period, decrease long-term complications, and facilitate forward compatibility with respect to exchange, recovery, and transplantation.

\section{References}

1. Saeed D, Sixt S, Albert A, Lichtenberg A. Minimally invasive off-pump implantation of HeartMate 3 left ventricular assist device. J Thorac Cardiovasc Surg. 2016;152:1446-7.

2. Sileshi B, Haglund NA, Davis ME, Tricarico NM, Stulak JM, Khalpey Z, et al. Inhospital outcomes of a minimally invasive off-pump left thoracotomy approach using a centrifugal continuous-flow LVAD. J Heart Lung Transplant. 2015;34:107-12.

3. Strueber M, Meyer AL, Feussner M, Ender J, Correia JC, Mohr FW. A minimally invasive off-pump implantation technique for continuous-flow LVADs: early experience. J Heart Lung Transplant. 2014;33:851-6. 